\title{
Analysis of beam propagation in optical fiber structures with a high step index
}

\author{
Krzysztof B. Zegadło ${ }^{*}$ and Mirosław A. Karpierz \\ Faculty of Physics, Warsaw University of Technology, Koszykowa 75, 00-662 Warszawa
}

Received December 16, 2009; accepted December 29, 2009; published December 31, 2009

\begin{abstract}
Microstructural fibers with high steps of the refractive index require special methods to calculate light propagating. In this paper three numerical methods describing beam propagation are compared. This comparison is shown for a two-core cylindrical waveguide.
\end{abstract}

In recent years we can observe very fast development of complex waveguide structures as microstructural fibers [1], photonic nanowires [2] and slot waveguides [3], because of their prospective applications. Microstructural fibers have special structure which allows to obtain many nonlinear phenomena. These fibers consist of a solid pure silica core surrounded by an air hole lattice in the cladding. Such construction provides many useful properties as ultra flattened dispersion, tailored mode area, broadband single-mode guidance, high numerical aperture, adjustable dispersion, high birefringence, large or ultra small effective areas, large nonlinearity, etc. In order to design and optimize such waveguides, good and fast numerical tools are needed. Most of algorithms consider only the weakly guiding case with simple boundary conditions, proper for small changes of the refractive index. However, in microstructural fibers a high step of the refractive index takes place. This work considers also the case of a huge step of the refractive index. There are compared three numerical, fast and simple approximation methods applied to the directional coupler with a big difference of the refractive index between cores and cladding (Fig. 1.).

The first examined method is the Beam Propagation Method (BPM) [4], relying on a numerical solution of Maxwell's equations. Usually, one assumes slowly varying envelope approximation, so the second derivative of amplitude over dz can be omitted when compared with the first derivative $(\mathrm{z}$ is the direction of beam propagation).

\footnotetext{
*E-mail: zegadlo@if.pw.edu.pl
}
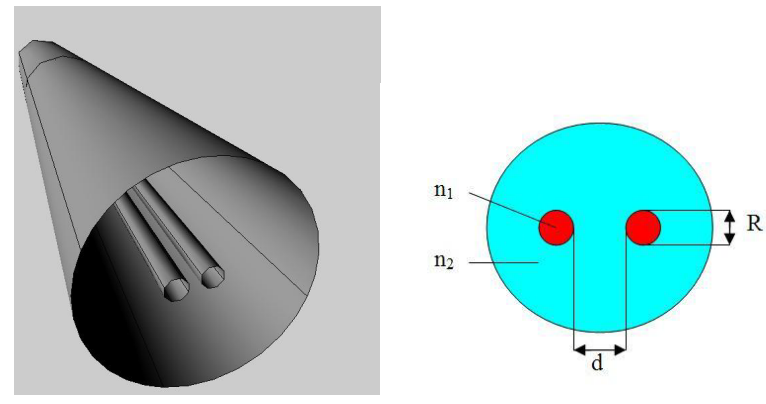

Fig. 1. Directional coupler consisting of two cylindrical cores surrounded by cladding

In the scalar version of the BPM, an electric field is polarized, in our case along the $\mathrm{x}$-axis and:

$$
\mathrm{E}_{x}=\mathrm{A}(x, y, z) e^{i \omega t-i \beta z}
$$

where $\beta$ is a constant. If the contrast ratio between indices of the core and cladding is very small (weakly guiding), one can treat it as a homogeneous medium case. It allows dropping out the term with a divergence of electric field $\mathrm{E}$ and obtaining a standard wave equation:

$$
\frac{\partial \mathrm{A}}{\partial z}=\frac{1}{2 i \beta}\left[\frac{\partial^{2}}{\partial x^{2}}+\frac{\partial^{2}}{\partial y^{2}}+\left(\frac{n^{2}(x, y) \omega^{2}}{c^{2}}-\beta^{2}\right)\right] \mathrm{A}
$$

All derivatives in this equation are substituted for finite differences. The BPM requires only the values of the E field in the initial step. In further steps the amplitude is calculated from the points of the previous step. Additionally, this method requires proper boundary conditions at the edge of a calculation frame. The most commonly used are Transparent Boundary Conditions (TBC) [5] and used in our simulations Perfect Matched Layer (PML) [6]. 
a)

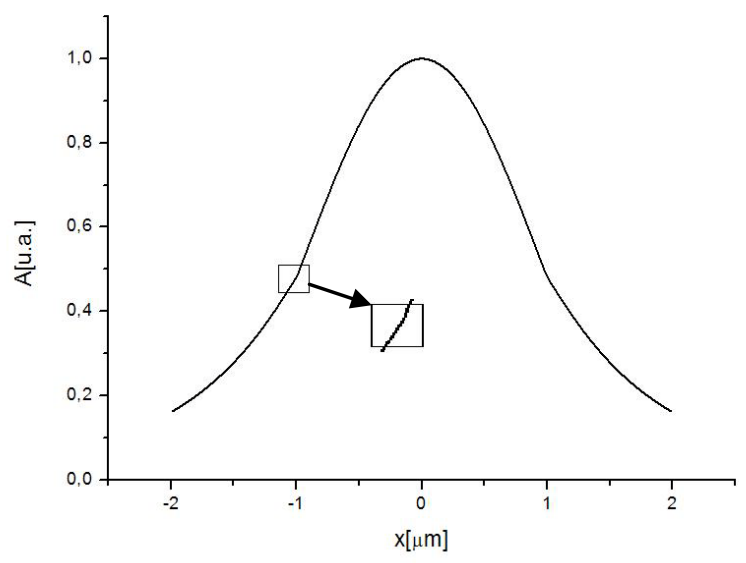

b)

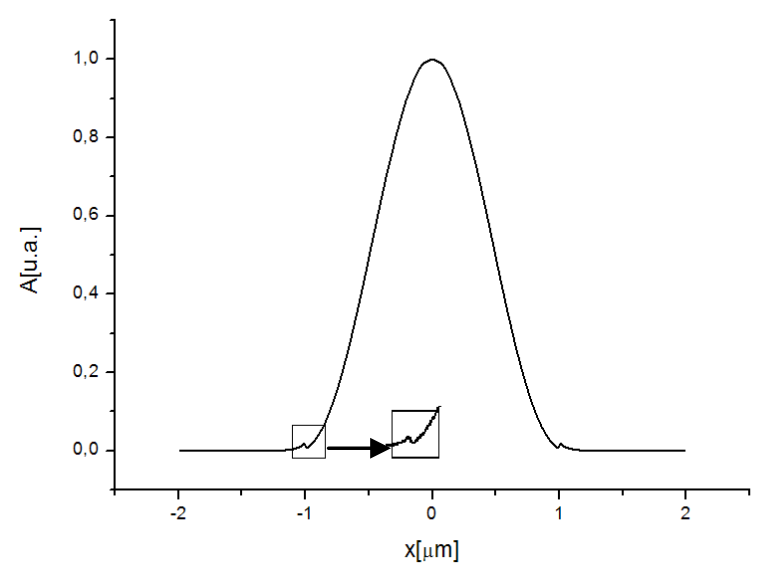

Fig. 2. Electric field profile in $\mathrm{HE}_{11}$ mode in a single core optical fiber with a radius $1 \mu m$ for a) low difference of indices $n_{1}-n_{2}=0.008$ and for b) high difference of indices $n_{1}-n_{2}=0.5$

This method gives a very good approximation for the weakly guiding case, but for higher contrast it is not proper because of the $\mathrm{E}$ field divergence on the bounds between cores and cladding. In order to satisfy the discontinuity of an electric field, one must apply proper boundary conditions. It can be clearly shown as an exact analytical solution (Fig. 2.) for $\mathrm{HE}_{11}$ in a single core optical fiber.

The second method is a coupled mode theory (CMT) [7], [8]. It treats Maxwell's equations in an analytical way considering the field as a superposition of waveguide modes. In the case of 2 cores, the refractive index of a neighbouring core is treated as a disturbance. This perturbation causes additional polarization, which is the source of a field excited in another core. One cylindrical core has an exact analytical solution (Bessel functions) and the full form of the field in two cylindrical cores structure has the form:

$$
E=\mathrm{A}_{1}(z) E_{1}(x, y) e^{i \omega t-i \beta z}+\mathrm{A}_{2}(z) E_{2}(x, y) e^{i \omega t-i \beta z},
$$

where $E_{1}, E_{2}$ - modes of separated waveguides, $\beta$ - the propagation constant. One has to calculate amplitudes for both modes as a function of the propagation distance:

$$
\frac{d}{d z} \mathrm{~A}_{m}=-i \kappa_{m m} \mathrm{~A}_{m}-i \kappa_{m n} \mathrm{~A}_{n}
$$

where $\mathrm{m}, \mathrm{n}=1,2$ and $m \neq n$. To solve these amplitudes one has to obtain the coupling constant of two modes:

$$
\kappa_{m n}=\frac{\omega \varepsilon_{0}}{\mathrm{P}} \int_{-\infty}^{\infty} \int_{-\infty}^{\infty} d x d y E_{m} \delta \varepsilon E_{n}
$$

where $\delta \varepsilon=n_{1}^{2}-n_{2}^{2}$ is perturbation in the second core, and $\mathrm{P}$ is a factor obtained by integrating the vector product of $E_{1}$ and $E_{2}$ over the whole calculation frame.

The third method reduces 3-dimensional BPM to 2D BPM by using the effective index method (Neff) [9]. Each core is divided into small pieces (Fig. 3.) and each of these pieces is substituted for a planar waveguide with a corresponding width. The dispersion equation must be solved and an effective refractive index is obtained for each of these pieces.
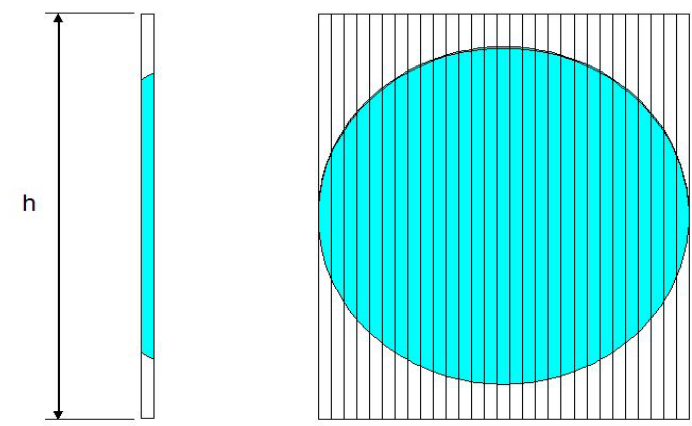

Fig. 3. Neff method applied to the cylindrical core

As a consequence of that, one obtains the distribution of the refractive index and one can create a twodimensional structure with such refractive index distribution. As it is obviously a 2D problem, the time of calculation radically decreases. 
a) $\Delta n=0.008$

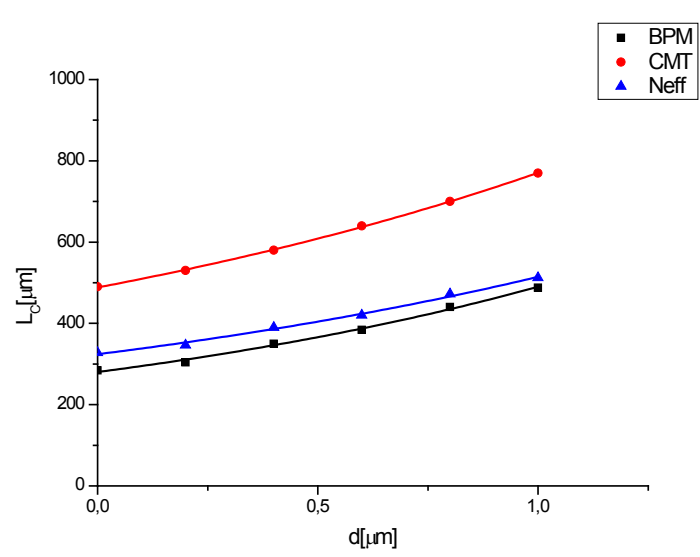

b) $\Delta n=0.1$

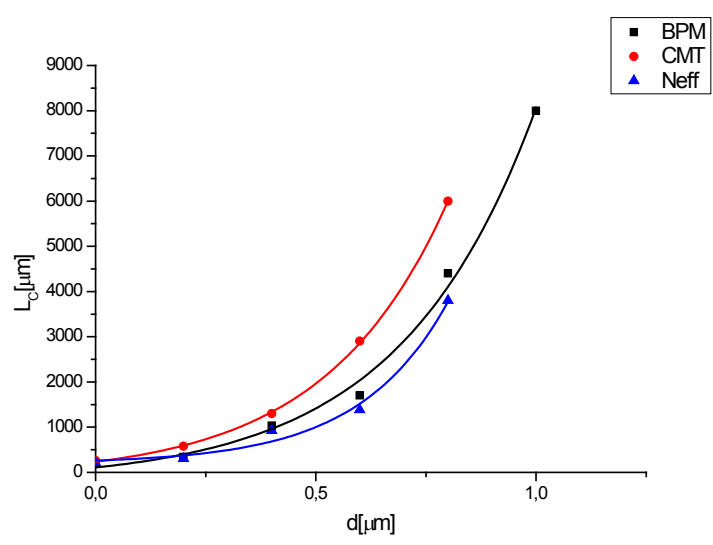

c) $\Delta n=0.5$

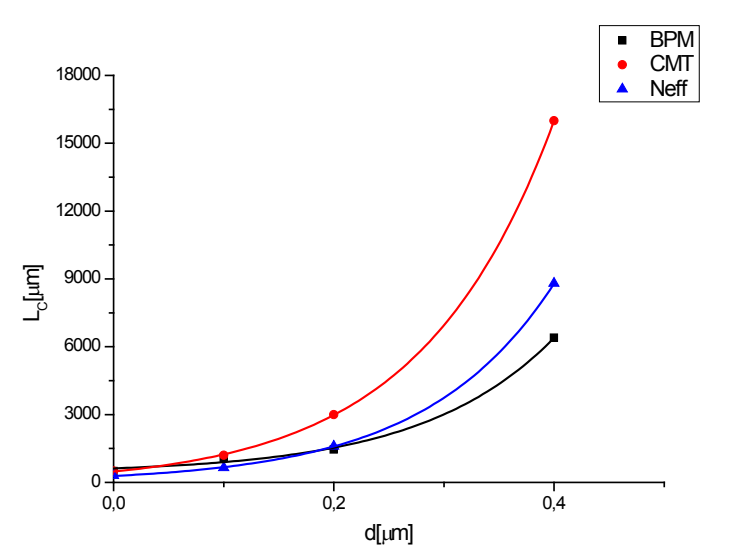

Fig. 4. Coupling length as a function of the distance between cores obtained with the help of 3 methods for the difference of refractive indices a) $\Delta n=0.008$, b) $\Delta n=0.1$ and c) $\Delta n=0.5$
The comparison of results obtained by these three methods is presented in Fig. 4. This comparison concerns coupling length $\mathrm{L}_{\mathrm{C}}$, which is defined as the distance on which the field in one core decreases from its maximal value to zero, because of switching to the second core, and then increases back to its maximal value. In CMT $\mathrm{L}_{\mathrm{C}}$ is given by:

$$
L_{C}=\frac{1}{\kappa_{12}}
$$

We show the comparison for three cases, when the difference of refractive index between cores and cladding is $\Delta n=n_{1}-n_{2}=0.008,0.1$ and 0.5 (Fig. 4.). One can see that the smallest differences in the coupling length are in the case of index difference $\Delta n=0.008$, so it is obviously weakly guiding. For a bigger value of $\Delta n$ the results obtained by these three methods are similar for very small distance between two cores (cores are close to each other), but for larger distances there is a big divergence between the results. One can observe it especially for $\Delta n=0.5$, which corresponds to silica cores surrounded by air. BPM and Neff do not yield the results so different from each other as CMT. Neff and CMT are several times faster than the 3D BPM algorithm. When the value of $\Delta n$ is not so large, one can use any of these two methods instead of 3D BPM. Also for a high step index with a very small distance between cores one can use CMT or Neff, but for longer distances one should compare all the three methods so as to obtain the most exact solution of the problem.

\section{References}

[1] J. M. Fini, Meas. Sci. Technol. 15, 1120-1128 (2004)

[2] R. Yan, D. Gargas, P. Yang, Nature Photonics 3, 569-576 (2009)

[3] A. H. J. Yang, Nature 457, 71-75 (2009)

[4] J. E. Midwinter, Optical Fibers for transmission (John Wiley, 1979).

[5] G. R. Hadley, Opt. Lett. 16, 624-626 (1991)

[6] J. P. Berenger, J. Comput. Phys. 114, 185-200 (1994)

[7] A. Yariv, IEEE J. Quantum Electron. QE-9, 919 (1973)

[8] W. Huang, J. Opt. Soc. Am. A 11, 963-983 (1994)

[9] J. C. Knight, T. A. Birks, P. J. Russel, J. P. de Sandro J. Opt. Soc. Am. A 15, 748-752 (1998) 